\title{
REVERSIBLE DILATED CARDIOMYOPATHY AFTER LOBECTOMY FOR PULMONARY SEQUESTRATION
}

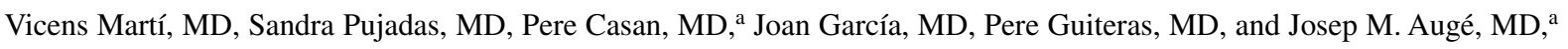 \\ Barcelona, Spain
}

Pulmonary sequestration is a congenital malformation characterized by a pulmonary lobe or pulmonary segment that receives blood supply directly from the aorta. Classically, 2 forms are described. The first is intralobar sequestration, in which the anomalous lung tissue is contained within the visceral pleura. The blood supply comes directly from the aorta, and normal venous drainage through the pulmonary veins goes to the left atrium. The second form is extralobar sequestration, in which the abnormal lung tissue is separated from the normal lung by its own visceral pleural investment. The blood supply generally derives either from the abdominal aorta or the pulmonary artery, and the venous drainage is through the azygos vein or portal system. ${ }^{1}$

The relevance of this pathologic condition derives from the difficulty in diagnosis and the risk of development of severe pulmonary and cardiovascular complications. Heart failure usually appears during the neonatal period or childhood and in most patients is associated with congenital cardiac defects. ${ }^{2,3}$ We report the case of an adult patient without structural cardiac abnormalities in whom a dilated cardiomyopathy caused by an intralobar pulmonary sequestration undiagnosed for many years reverted after lobectomy.

Clinical summary. A 30-year-old woman with no pathologic antecedents was seen in our center for increasing shortness of breath of 3 years' duration. Physical examination revealed a systolic ejection murmur. Results of pulmonary auscultation, electrocardiography, and chest x-ray films were normal. An echocardiogram showed dilatation of the left ventricle and a mild reduction of the left ventricular ejection fraction (Table I). No regurgitations or blood flow accelerations were detected with Doppler ultrasonography. Biochemical and immunologic blood test results were within normal limits. Systemic diseases were

From the Department of Cardiology and Cardiac Surgery and the Service of Pneumology, ${ }^{a}$ Hospital de la Santa Creu i Sant Pau, Barcelona, Spain.

J Thorac Cardiovasc Surg 2001;121:1001-2

Copyright (ㄷ 2001 by The American Association for Thoracic Surgery

$0022-5223 / 2001 \$ 35.00+0 \quad \mathbf{1 2 / 5 4 / 1 1 2 3 2 0}$

doi: $10.1067 / \mathrm{mtc} .2001 .112320$
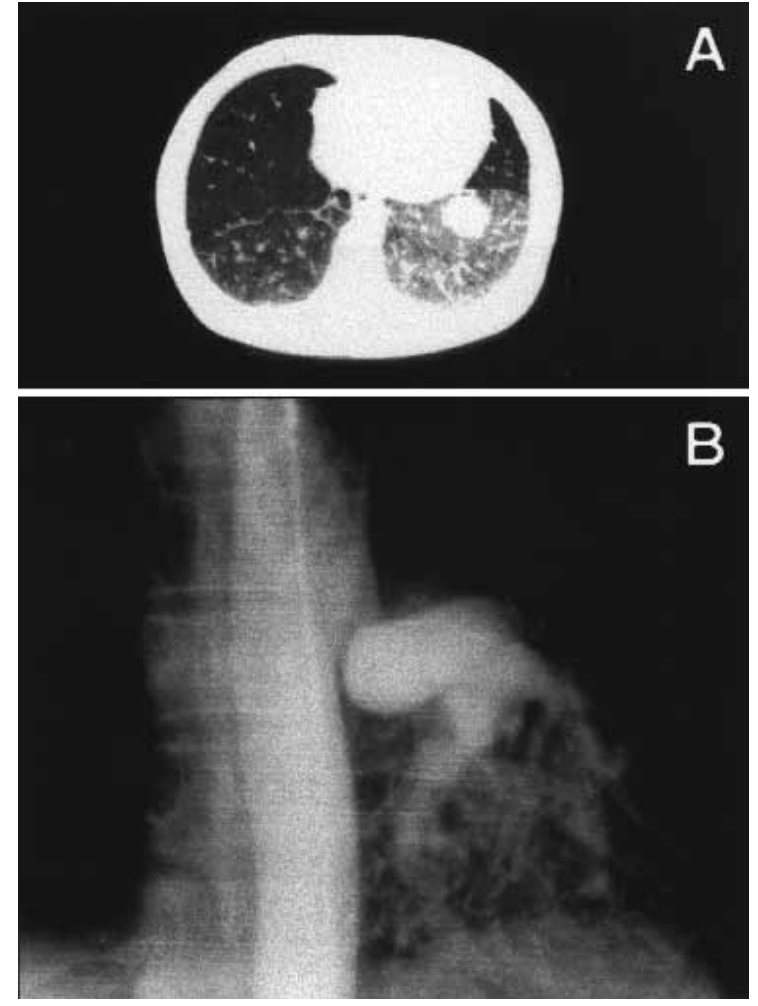

Fig 1. A, Computed tomography revealed a pulmonary sequestration with a large anomalous vessel inside. B, Aortography demonstrates an aberrant artery arising from the supradiaphragmatic descending aorta. It supplies blood to the lower left lobe pulmonary sequestration.

ruled out. The patient was not taking any cardiotoxic drug. She was given a diagnosis of idiopathic dilated cardiomyopathy. Oral diuretic and vasodilator treatment was given, and follow-up was recommended. Seven years later, she was admitted for increased shortness of breath and productive cough. Spirometric analysis performed in the pneumology service showed a mild air flow limitation. Allergic skin test responses were negative. A control echocardiogram did not show significant changes in the 
Table I. Echocardiographic measurements before and after resection of a pulmonary sequestration

\begin{tabular}{lcrrr}
\hline & & \multicolumn{3}{c}{ After operation } \\
\cline { 2 - 5 } & Baseline & 8 mo & 16 mo & 20 mo \\
\hline Heart rate (beats/min) & 78 & 74 & 72 & 72 \\
LV end-diastolic diameter (mm) & 65 & 58 & 54 & 48 \\
LV ejection fraction (\%) & 58 & 59 & 65 & 62 \\
Index of LV mass & 168 & 115 & 110 & 73 \\
\hline
\end{tabular}

$L V$, Left ventricular.

ventricular size or function. A diagnosis of bronchial asthma was given, and inhaled bronchodilators were prescribed. Three years later, in a follow-up chest x-ray film, a mass in the lower left lung field was observed. Computed tomographic scanning with contrast showed an anomalous systemic vascularization directly from the thoracic aorta to the lower left lobe (Fig 1, A) and normal venous drainage to the left atrium through the pulmonary veins. Aortography demonstrated the lobar inferior artery arising from the thoracic descending aorta that irrigated the left lower lobe (Fig 1,B). Four months later, a left lower lobectomy was performed without postoperative complications. During the follow-up period, progressive reduction of the cardiac mass and left ventricular size and improvement of the left ventricular function were seen (Table I). Twenty months after the operation, the echocardiograpic results were normal, and the patient was asymptomatic without drugs.

Discussion. The most common finding leading to the diagnosis of pulmonary sequestration are the pulmonary symptoms (cough, recurrent infections, dyspnea, or hemoptysis), an abnormal x-ray film finding, or both. ${ }^{1}$ Cardiovascular symptoms caused by pulmonary sequestration in the absence of associated intracardiac abnormalities are rare, with the most commonly reported being congestive heart failure, which usually appears in the neonatal period or in childhood..$^{2,45}$ In adults the appearance of cardiovascular complications ascribed to the sequestrum is very uncommon. This may be because the majority of patients are given diagnoses and treated before heart failure develops.

Cardiac complications may be caused by the high cardiac output, the diastolic volume overload to the left ventricle caused by the left-to-left shunt through the pulmonary sequestration, or both. ${ }^{1,6}$ The magnitude of the shunt may influence the moment of appearance of cardiac symptoms. The presence of a high shunt during childhood may contribute to the early development of overt cardiac failure, which may lead to early diagnosis and surgical treatment. However, the sequestrum may be overlooked if the shunt is small or there are no associated cardiac abnormalities or pulmonary symptoms. In this case the appearance of cardiovascular complications can be time dependent. However, the magnitude of the shunt through the sequestrum may increase as the patient grows older. ${ }^{1}$ To our knowledge, only 2 cases of cardiac alterations have been reported in adults. Solit and colleagues ${ }^{6}$ described a 23 -year-old patient with an intralobar pulmonary sequestration in whom the preoperative hemodynamic study showed a high cardiac output without the signs of heart failure; the diagnosis of sequestrum was made after a routine chest $\mathrm{x}$-ray film. Fabre and colleagues ${ }^{7}$ described a 25 -year-old patient with an undiagnosed pulmonary sequestration associated with aortic valve stenosis who had cardiomegaly and congestive heart failure, which were attributed to aortic valvulopathy. The patient's condition improved after removal of the sequestrum. Our patient did not have any structural cardiac abnormality that could provoke heart failure. Because the pulmonary sequestration remained undiagnosed for many years, a diagnosis of idiopathic dilated cardiomyopathy was erroneously made. Treatment with vasodilators and diuretics probably avoided overt heart failure during the follow-up period. Pulmonary sequestration was diagnosed as a result of pulmonary symptoms. It was then suspected that the symptoms could have been caused by the left-to-left shunt through the sequestrum. However, secondary dilated cardiomyopathy could only be diagnosed after the evidence of normalization of the cardiac mass and the ventricular size and function after lobectomy.

Progressive and irreversible cardiac dilatation has been observed in other clinical situations with a diastolic overload volume over a long period of time. ${ }^{8}$ Therefore, early diagnosis and treatment of pulmonary sequestration is important before irreversible cardiac damage occurs. The presence of pulmonary sequestration should be considered in the differential diagnosis of dilated cardiomyopathy.

Received for publication July 28, 2000; accepted for publication Oct 16, 2000.

Address for reprints: Vicens Martí, MD, Department of Cardiology and Cardiac Surgery, Hospital de la Santa Creu i Sant Pau, Av Sant Antoni M. Claret 167, 08025-Barcelona, Spain (E-mail: 18461vmc@comb.es).

\section{REFERENCES}

1. Khalil KG, Kilman JW. Pulmonary sequestration. J Thorac Cardiovasc Surg 1975;70:927-37.

2. White J, Donahoo J, Ostrow P, Murphy J, Haller J. Cardiovascular and respiratory manifestations of pulmonary sequestration in childhood. Ann Thorac Surg 1974;18:286-94.

3. Flye MW, Conley M, Silver D. Spectrum of pulmonary sequestration. Ann Thorac Surg 1976;49:478-82.

4. Ramson J, Norton J, Williams G. Pulmonary sequestration presenting as congestive heart failure. J Thorac Cardiovasc Surg 1978;76:378-80.

5. Levine MM, Nudel DB, Gootman N, Wolpowitz A, Wisoff G. Pulmonary sequestration causing congestive heart failure in infancy: a report of two cases and review of the literature. Ann Thorac Surg 1982;34:581-5.

6. Solit RW, Frainow W, Wallace S, Cohn HE. The effect of intralobar pulmonary sequestration on cardiac output. J Thorac Cardiovasc Surg 1964;49:844-53.

7. Fabre OH, Porte HL, Godart FR, Rey C, Wurtz AJ. Long-term cardiovascular consequences of undiagnosed pulmonary sequestration. Ann Thorac Surg 1998;65:1144-6.

8. Yamazaki J, Igarashi M, Nakata M, Okamoto K, Hosoi H, Morishita T, et al. Estimating myocardial damage and the need for surgery in patients with valvular heart disease by Tl-201 SPECT. Clin Nucl Med 1996;21:855-63. 